Surgery for

Congenital

Heart Disease

\title{
Regional low-flow perfusion provides comparable blood flow and oxygenation to both cerebral hemispheres during neonatal aortic arch reconstruction
}

Dean B. Andropoulos, MD

Stephen A. Stayer, $M D^{\mathrm{a}}$

E. Dean McKenzie, MD, FACS ${ }^{b}$

Charles D. Fraser, Jr, MD, FACS ${ }^{b}$
From the Divisions of Pediatric Cardiovascular Anesthesiology ${ }^{\mathrm{a}}$ and Congenital Heart Surgery, ${ }^{\mathrm{b}}$ Texas Children's Hospital and Baylor College of Medicine, Houston, Tex.

Received for publication Feb 10, 2003; revisions requested Feb 10, 2003; revisions received May 16, 2003; accepted for publication June 17, 2003.

Address for reprints: Dean B. Andropoulos, MD, Texas Children's Hospital, Pediatric Cardiovascular Anesthesiology, 6621 Fannin, WT19345H, Houston TX 77030-2399 (E-mail: dra@bcm.tmc.edu).

J Thorac Cardiovasc Surg 2003;126:1712-7

Copyright $(2) 2003$ by The American Association for Thoracic Surgery

$0022-5223 / 2003 \$ 30.00+0$

doi:10.1016/S0022-5223(03)01027-4
Objective: The aim of this study was to measure cerebral oxygenation, cerebral blood volume index, and cerebral blood flow velocity values in both cerebral hemispheres before, during, and after regional low-flow cerebral perfusion for neonatal aortic arch reconstruction and to test the hypothesis that cerebral blood volume index measured by near infrared spectroscopy correlates with cerebral blood flow velocity measured by transcranial Doppler ultrasonography.

Methods: Bilateral near infrared spectroscopy and transcranial Doppler ultrasonography sensors were placed, and values were recorded immediately before, during, and after regional low-flow cerebral perfusion at $18^{\circ} \mathrm{C}$. Cerebral oxygen saturations, cerebral blood flow velocities, and cerebral blood volume index values were compared by Mann-Whitney $U$ test. Correlations between values of cerebral blood volume index and cerebral blood flow velocity were tested with Spearman rank order correlation.

Results: Twenty patients were studied. Median cerebral oxygen saturations for the right and left sides were $95 \%$ and $95 \%$ before regional low-flow cerebral perfusion, 95\% and $87 \%$ during regional low-flow cerebral perfusion $(P=.054)$, and $93 \%$ and 94\% after regional low-flow cerebral perfusion. Median cerebral blood flow velocity values did not change during regional low-flow cerebral perfusion. Cerebral blood volume index exhibited a poor correlation with cerebral blood flow velocity.

Conclusions: Regional low-flow cerebral perfusion provides comparable blood flows and oxygenation to both cerebral hemispheres. Transcranial Doppler ultrasonography is recommended as a corroborative method with near-infrared spectroscopy to guide flow during regional low-flow cerebral perfusion, because cerebral blood volume index does not correlate with cerebral blood flow velocity.

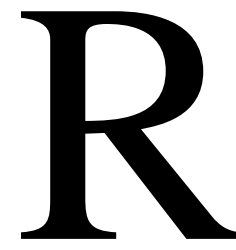

egional low-flow cerebral perfusion (RLFP) has gained increasing use as a perfusion technique to avoid or limit deep hypothermic circulatory arrest (DHCA) for the Norwood operation for hypoplastic left heart syndrome and other aortic reconstruction operations in the newborn period. ${ }^{1-3}$ With this technique a 3- to 4-mm polytetrafluoroethylene (PTFE) graft is anastomosed to the right innominate artery and serves as aortic inflow during bypass. During aortic recon- 
struction, the brain and right upper extremity only are perfused through the graft, with the brachiocephalic vessels and the descending thoracic aorta snared to achieve a bloodless field. Thus the brain receives direct unilateral perfusion through the right common carotid artery and right vertebral artery. Previous studies ${ }^{2,4}$ have used neurophysiologic monitoring of the right side of the brain (near-infrared spectroscopy [NIRS] and transcranial Doppler ultrasonography [TCD]) to demonstrate adequate cerebral blood flow velocity (CBFV), blood volume, and cerebral oxygenation during RLFP and to guide bypass flow rates. It has been assumed that the circle of Willis in the neonate is intact and that adequate blood flow is provided across it to the left side of the brain during RLFP. However, this assumption has been questioned recently, ${ }^{2}$ and there have been no reports of measurement of blood flow velocity and oxygenation in the left cerebral hemisphere during RLFP. Another assumption in previous work is that the cerebral blood volume index $(\mathrm{CBVI})^{2,5}$ as measured by NIRS correlates with cerebral blood flow (CBF) and can be used to guide bypass flow during RLFP. ${ }^{2}$

The purpose of this study was to measure regional cerebral oxygenation index $\left(\mathrm{rSO}_{2} \mathrm{i}\right), \mathrm{CBVI}$, and $\mathrm{CBFV}$ to both cerebral hemispheres before, during, and after RLFP. Our hypothesis was that RLFP would provide equal blood flows and oxygenation to both cerebral hemispheres. The secondary purpose of the study was to test the hypothesis that CBVI, as measured indirectly by NIRS, correlates with CBFV measured directly by TCD. The strength of association between CBVI and CBFV was tested to determine whether TCD monitoring was necessary or the same information could be derived from NIRS alone.

\section{Methods}

After institutional review board approval, infants undergoing aortic arch reconstruction were studied. Patients undergoing the Norwood operation for hypoplastic left heart syndrome and those undergoing aortic arch advancement for interrupted or hypoplastic aortic arch were studied if a period of RLFP was planned.

The anesthetic technique consisted of fentanyl (100-200 $\mu \mathrm{g} /$ $\mathrm{kg}$ ), midazolam (0.3-1.0 mg/kg), and pancuronium or vecuronium. Isoflurane $(<1 \%)$ was used to supplement the anesthetic before and during bypass. Arterial pressure monitoring consisted of an umbilical or femoral arterial catheter. In addition, a catheter was placed when possible in the left radial artery for patients undergoing the Norwood operation and the right radial artery for patients undergoing aortic arch advancement.

Cerebral physiologic monitoring included NIRS (INVOS 5100; Somanetics Corp, Troy, Mich) to measure $\mathrm{rSO}_{2} \mathrm{i}$ with bilateral sensors applied to the forehead just to the right and left of midline. This method uses near infrared light at 724- and 812-nm wavelengths to measure the absorption spectra of the total hemoglobin and deoxyhemoglobin in the frontal cerebral cortex. ${ }^{6}$ A shallow detector subtracts light absorbed by the skull and soft tissues, and a deep detector measures light absorbed by an area in the frontal cortex in the light path. A percentage $\mathrm{rSO}_{2} \mathrm{i}$ is displayed, which is derived as the ratio of oxyhemoglobin to total hemoglobin $\times$ $100 \%$ :

(Total hemoglobin signal at $812 \mathrm{~nm}$

$$
\frac{- \text { Deoxyhemoglobin signal at } 724 \mathrm{~nm})}{\text { Total hemoglobin signal at } 812 \mathrm{~nm}} \times 100 \%=\mathrm{rSo}_{2} \mathrm{i}
$$

In addition, the INVOS 5100 displays an index of near-infrared signal strength at $812 \mathrm{~nm}$, which is a dimensionless number from -50 to +50 . The -50 value is set by internal calibration by the monitor at startup before patient measurement. This signal strength is proportional to the total hemoglobin in the light path. If the hemoglobin concentration is not changing, this signal strength should be proportional to the cerebral blood volume (CBV) in the light path, giving rise to the designation $C B V I{ }^{7}$

Bilateral transcranial Doppler pulsed-wave ultrasonography (EME Companion; Nicolet Biomedical Inc, Madison, Wis) of the right and left middle cerebral arteries was used to measure CBFV. A 2-Mhz probe was placed over the right and left temporal areas, and depth of sample volume and angle of insonation were adjusted until a maximal bidirectional CBFV signal was obtained whenever possible, signifying sampling at the bifurcation of the middle and anterior cerebral arteries. ${ }^{8}$ When a bidirectional signal was not obtainable, the transducer was adjusted until the maximal signal above baseline (toward the transducer) was obtained in the middle cerebral artery. Mean sampling depth was $40 \pm 6 \mathrm{~mm}$. The depth of the sample volume was identical on both sides for all patients at all data points. In this manner we ascertained that the same area was being sampled on both sides of the brain. The mean CBFV and Doppler spectrum were displayed and recorded.

For patients undergoing the Norwood operation, bypass was initiated through an $8 \mathrm{~F}$ or $10 \mathrm{~F}$ aortic cannula inserted into the distal end of a 3- or 3.5-mm PTFE graft, with the proximal end anastomosed into the distal right innominate artery or proximal right subclavian artery. Monitoring of CBFV during innominate artery occlusion for graft placement ensured adequate right-sided cerebral flow during this period. Patients with interrupted aortic arch had a second arterial cannula placed in the patent ductus arteriosus to perfuse the lower body.

Bypass was instituted at a flow of 150 to $200 \mathrm{~mL} /(\mathrm{kg} \cdot \mathrm{min})$. Phenoxybenzamine $(0.25 \mathrm{mg} / \mathrm{kg})$ was administered to all patients undergoing the Norwood operation on initiation of bypass. Phenoxybenzamine $(0.25-1 \mathrm{mg} / \mathrm{kg})$ or phentolamine hydrochloride (0.3-1 mg/kg, INN: phentolamine) was administered to all other patients on bypass to achieve a mean arterial pressure of 30 to 40 $\mathrm{mm} \mathrm{Hg}$ at a minimum flow of $150 \mathrm{~mL} /(\mathrm{kg} \cdot \mathrm{min})$ throughout the duration of bypass. Extracorporeal cooling to a nasopharyngeal temperature of $18^{\circ} \mathrm{C}$ was achieved through no less than 20 minutes. The target hematocrit was $25 \%$ to $30 \%$ during the period of hypothermia. The $\mathrm{pH}$-stat blood gas strategy was used during all phases of the bypass period. For patients undergoing the Norwood operation, a brief period ( $<10$ minutes) of circulatory arrest was used during atrial septectomy. Then RLFP was used for the aortic reconstruction as follows. Snares were placed around the base of the right innominate, left common carotid, and left subclavian arteries and around the descending thoracic aorta distal to the coarctation. Perfusion was then instituted through the PTFE graft to the right innominate artery only during the aortic reconstruction. 
TABLE 1. Patient bypass data $(n=20)$

\begin{tabular}{|c|c|c|c|}
\hline Parameter & Before RLFP & During RLFP & After RLFP \\
\hline Right radial artery pressure $(\mathrm{mm} \mathrm{Hg}, \mathrm{n}=11)$ & $35 \pm 7$ & $33 \pm 7$ & $34 \pm 4$ \\
\hline Left radial artery pressure $(\mathrm{mm} \mathrm{Hg}, \mathrm{n}=7)$ & $31 \pm 3$ & $23 \pm 9^{*} \dagger$ & $36 \pm 6$ \\
\hline $\begin{array}{l}\text { Mean arterial pressure in umbilical or femoral } \\
\text { artery }(\mathrm{mm} \mathrm{Hg})\end{array}$ & $31 \pm 4$ & $11 \pm 3^{*}$ & $35 \pm 5$ \\
\hline Bypass flow (mL/ [kg . min]) & $175 \pm 45$ & $64 \pm 15^{*}$ & $170 \pm 28$ \\
\hline Hematocrit $(\%)$ & $27 \pm 2 \ddagger$ & $25 \pm 2$ & $25 \pm 2$ \\
\hline $\mathrm{pH}$ (temperature corrected) & $7.36 \pm 0.06$ & $7.41 \pm 0.07^{*}$ & $7.37 \pm 0.04$ \\
\hline $\mathrm{PcO}_{2}(\mathrm{~mm} \mathrm{Hg}$, temperature corrected) & $40 \pm 5$ & $36 \pm 6^{*}$ & $39 \pm 4$ \\
\hline Lactate $(\mathrm{mmol} / \mathrm{L})$ & $1.9 \pm 0.5$ & $2.1 \pm 0.5 \S$ & $2.7 \pm 0.8 \|$ \\
\hline Calculated base deficit & $-1.2 \pm 1.7$ & $-0.2 \pm 2.3$ & $-0.7 \pm 1.8$ \\
\hline
\end{tabular}

Results are expressed as mean \pm SD. Right radial artery pressure was measured only in patients with a right radial catheter, and left radial artery pressure was measured only in those with a left radial catheter.

${ }^{*} P<.05$ for RLFP versus before or after RLFP by 1-way repeated measures analysis of variance.

$\dagger P=.03$ versus right radial artery pressure during RLFP by $t$ test.

$\ddagger P<.05$ before RLFP versus during and after RLFP.

$\S P<.05$ during RLFP versus after RLFP.

$\| P<.05$ after RLFP versus before RLFP.

\section{TABLE 2. Patient demographic data $(\mathbf{n}=\mathbf{2 0})$}

Age (d, median and interquartile range) $6(4-9)$

Weight $(\mathrm{kg}$, median and interquartile $3.4(2.7-3.6)$ range)

Baseline hematocrit $(\%$, median and interquartile range)

Norwood operation (No.)

Aortic arch advancement (No.)

Bypass time (min, median and interquartile range)

Myocardial ischemic time (min, median and interquartile range)

DHCA time (min, median and interquartile range)

RLFP time (min, median and interquartile range)

30-d in-hospital deaths (No.)

New neurologic deficits (No.) body bypass at deep hypothermic temperatures $\left(18^{\circ} \mathrm{C}\right)$ immediately preceding RLFP, (2) during RLFP at $18^{\circ} \mathrm{C}$ after adjustment of bypass flow rate to achieve mean CBFV values within $10 \%$ of baseline (measured at full-flow hypothermic bypass during period 1 just preceding) values for both, and (3) within 5 minutes after aortic reconstruction during full-flow bypass to the whole body at $18^{\circ} \mathrm{C}$ after steady-state $\mathrm{rSO}_{2} \mathrm{i}$ and $\mathrm{CBFV}$ values were achieved before rewarming. Data collected at the three bypass times included bypass flow rate; bilateral $\mathrm{rSO}_{2} \mathrm{i}, \mathrm{CBVI}$, and $\mathrm{CBFV}$ values; mean pressure in the radial and umbilical or femoral arteries; temperature-corrected blood gas values; hematocrit; calculated base excess; and plasma lactate. Normally distributed data are expressed as mean $\pm \mathrm{SD}$, and nonnormally distributed data are expressed as median with $25 \%$ to $75 \%$ interquartile ranges. Bypass data in Table 1 were normally distributed and were compared by $t$ test and 1-way repeated measures analysis of variance. Other data were not normally distributed, so the Mann-Whitney $U$ test was used to compare right- and left-sided values of $\mathrm{rSO}_{2} \mathrm{i}, \mathrm{CBVI}$, and CBFV. Kruskal-Wallis 1-way analysis of variance on ranks was used to compare values among the three times for each cerebral hemisphere. Spearman rank order correlation was used to correlate CBFV values with their corresponding CBVI values at identical times for each cerebral hemisphere (SPSS Inc, Chicago, Ill).

Outcome follow-up consisted of a medical record review for the following: 30-day postoperative or in-hospital mortality, late mortality after 30 days, and neurologic deficits recorded by each patient's primary cardiologist during postoperative outpatient clinic visits.

\section{Results}

Twenty patients were studied. Eleven patients underwent the Norwood operation for hypoplastic left heart syndrome, and 9 underwent aortic arch advancement with other intracardiac procedures for severely hypoplastic or interrupted aortic arch. Patient bypass data are displayed in Table 1, and 
TABLE 3. Bilateral neurologic monitoring data $(n=20)$

\begin{tabular}{|c|c|c|c|c|c|c|c|c|}
\hline & \multicolumn{2}{|c|}{ Baseline } & \multicolumn{2}{|c|}{ Before RLFP } & \multicolumn{2}{|c|}{ During RLFP } & \multicolumn{2}{|c|}{ After RLFP } \\
\hline & Right & Left & Right & Left & Right & Left & Right & Left \\
\hline $\mathrm{rSO}_{2} \mathrm{i}(\%)$ & $55(43-63)$ & $51(46-59)$ & $95(91-95)$ & $95(83-95)$ & $95(91-95)$ & $87(81-95)^{*}$ & $93(86-95)$ & $94(79-95)$ \\
\hline CBFV $(\mathrm{cm} / \mathrm{s})$ & $18(14-23)$ & $18(13-24)$ & $19(15-23)$ & $19(14-23)$ & $19(17-25)$ & $20(14-30)$ & $17(14-20)$ & $16(13-23)$ \\
\hline CBVI & $15(9-17)$ & $18(12-30)$ & $7(-6-16)$ & $8(-16-23)$ & $6(1-18)$ & $14(0-30)$ & $9(1-17)$ & $16(-2-27)$ \\
\hline
\end{tabular}

Results are expressed as median with $25 \%$ to $75 \%$ interquartile range deviation.

${ }^{*} P=.054$ for right versus left side during RLFP, Mann-Whitney $U$ test.

patient demographic data are displayed in Table 2. During RLFP, pressure in the radial artery was significantly lower in those patients with a left radial catheter than in those with a right radial catheter. Neurologic monitoring data are reported in Table 3.

Paired values for CBFV were the same before, during, and after RLFP. The median $\mathrm{rSO}_{2} \mathrm{i}$ values were $8 \%$ lower on the left side during RLFP $(P=.054$, Mann-Whitney $U$ test $)$. Five of 20 patients had a difference of at least $10 \%$ in $\mathrm{rSO}_{2} \mathrm{i}$ during RLFP, always with the left side lower than the right. The largest single discrepancy was in a patient with $92 \%$ on the right and $68 \%$ on the left; this was the only patient with $\mathrm{rSO}_{2} \mathrm{i}$ less than 70\%. Eight of 20 patients had a difference of at least $25 \%$ in CBFV during RLFP, with the right side greater than the left in 4 and the left greater than the right in 4. Right-sided CBFV during RLFP did not correlate with RLFP flow rate $(P>.05$ by Spearman Rank Order Correlation; Figure 1). Poor correlation between individual CBVI and CBFV values is shown in Figure $2(P>.05$, Spearman rank order correlation). All paired values were studied together, because hematocrit demonstrated little change during the study period (27\% before vs $25 \%$ during and after RLFP), and separate analysis revealed no differences.

There were no in-hospital deaths within 30 days of the operation and no new neurologic deficits in this group of patients. One patient was discharged to home at the age of 21 days after a Norwood operation and died of Klebsiella pneumoniae sepsis at the age of 28 days. Another patient who underwent the Norwood operation died in the hospital at the age of 47 days of multiorgan system failure related to bacterial sepsis. A brain magnetic resonance imaging study done 2 weeks before was unremarkable.

\section{Discussion}

This study demonstrates that RLFP, when flow rate is guided by TCD, results in adequate blood flow and oxygenation to both cerebral hemispheres. We believe that the median difference in $\mathrm{rSO}_{2} \mathrm{i}$ between right and left hemispheres of $8 \%$ during RLFP is not clinically significant. Discrepancies between hemispheres in individual patients suggest that bilateral monitoring, especially of $\mathrm{rSo}_{2} \mathrm{i}$, may be useful. An alternative to bilateral monitors would be to place the NIRS sensor on the left side of the forehead, because this side appears to have a lower $\mathrm{rSO}_{2} \mathrm{i}$ in some patients. The TCD probe could be placed on the right temporal window; thus both hemispheres could be monitored with a single set of sensors. The lowest left hemisphere $\mathrm{rSO}_{2} \mathrm{i}$ of $68 \%$ was still significantly higher than the baseline preoperative $\mathrm{rSO}_{2} \mathrm{i}$ for that patient. Thus the usual "luxury cerebral perfusion" with $\mathrm{rSO}_{2} \mathrm{i}$ greater than $90 \%$ seen at deep hypothermia may not always be consistent during RLFP; however, the lower $\mathrm{rSO}_{2} \mathrm{i}$ on the left side would not appear to put the patients at risk for cerebral hypoxemia if RLFP is performed according to this protocol.

There are several possible explanations for the decreased $\mathrm{rSO}_{2} \mathrm{i}$ in the left cerebral hemisphere during RLFP. Although the circle of Willis is expected to be intact without stenoses in newborn patients, variations from the classic anatomic description are frequent, occurring in as many as $40 \%$ of adult autopsy specimens. ${ }^{9,10}$ In a study of color Doppler patterns in 53 patients, $10 \%$ of healthy term neonates showed deviations from normal flow patterns. ${ }^{11}$ In our patients, preferential flow to the right side or a nonintact circle of Willis can be argued against because CBFV on the left side was unchanged or slightly increased in all 5 patients with decreased $\mathrm{rSO}_{2} \mathrm{i}$ relative to the pre-RLFP period. In addition, we performed a test occlusion of the right innominate artery just before the anastomosis of the proximal end on the PTFE graft and demonstrated adequate $\mathrm{CBFV}$ and $\mathrm{rSO}_{2} \mathrm{i}$ to both cerebral hemispheres before proceeding with RLFP. Another possibility would be monitoring artifacts or failures; however, the NIRS system signal was adequate during all of the measurements, and the left side was always the side affected when there was a significant discrepancy in $\mathrm{rSO}_{2} \mathrm{i}$. The baseline $\mathrm{rSO}_{2} \mathrm{i}$ on the left was at least $10 \%$ less than the right before RLFP in 4 of the 5 patients who had a discrepancy during RLFP. Baseline $\mathrm{rSo}_{2} \mathrm{i}$ before incision in these patients was within $7 \%$ in 3 of these 4 patients, suggesting that in most cases the issues with unequal oxygenation began with the institution of full bypass, not RLFP itself. Only 1 of the 5 patients had unequal cerebral oxygenation exclusively during the period of RLFP. One explanation for both the lower $\mathrm{rSO}_{2} \mathrm{i}$ values on the left side for individual patients is that our practice in this institution is to retract the left innominate vein during bypass in newborns with a silicone elastomer vessel loop. This 


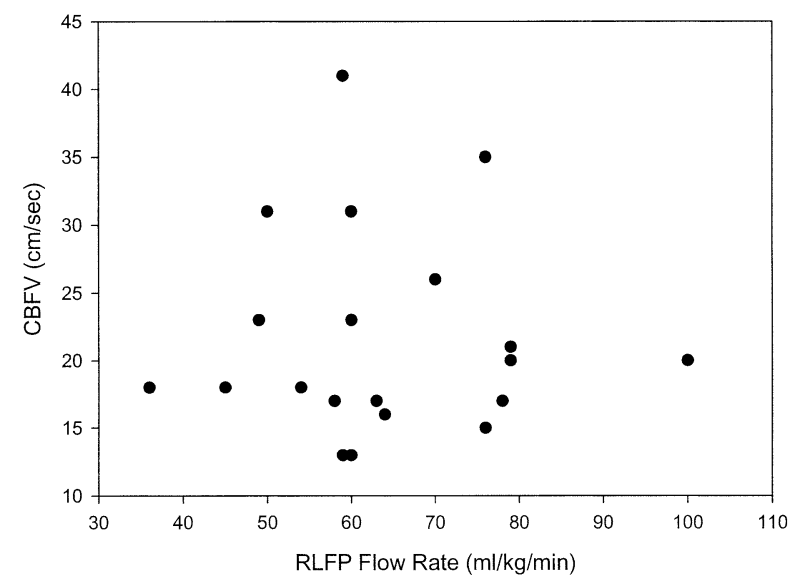

Figure 1. RLFP flow rate versus mean CBFV during RLFP. $P>.05$ by Spearman rank order correlation.

maneuver could partially obstruct this vessel and decrease cerebral venous drainage. In addition, the patient's head is always turned $45^{\circ}$ to $60^{\circ}$ to the left. These maneuvers could result in accumulation of a volume of slowly desaturating venous blood in the left cerebral hemisphere. Because the venous/arterial blood ratio ranges from $75: 25$ to $85: 15,{ }^{12}$ this would explain the lower saturations seen in the left cerebral hemisphere in some patients.

Bilateral monitoring could theoretically detect decreased cerebral oxygenation related to anatomic problems and allow for planning alternative surgical or bypass techniques. Bilateral monitoring of adult patients during aortic arch surgery has been described as potentially averting neurologic disaster when significant desaturation on one side was detected, allowing rapid alteration of surgical approach to normalize $\mathrm{rSO}_{2} \mathrm{i}$ on both sides. ${ }^{13}$

In a previous report, ${ }^{4}$ we described the use of TCD as the primary determinant of necessary bypass flow during RLFP. The added value of TCD relative to NIRS alone is avoidance of cerebral hyperperfusion. In that study, more than a third of patients had $\mathrm{rSO}_{2} \mathrm{i}$ values of $95 \%$ during deep hypothermia, which is the maximum reading by the monitor, leaving them potentially vulnerable to cerebral hyperperfusion when flow is adjusted to achieve this saturation. Radial artery pressure and a predetermined flow rate $(25 \%$ of normal full-bypass flow) have been used to guide RLFP flow, but both have poor correlation with CBFV. Another reason to add TCD is that the frontal lobe and the territory of the anterior and middle cerebral arteries can be monitored so that as much as $70 \%$ of one hemisphere can potentially be assessed. ${ }^{11}$ CBVI was described by Pigula and cowork$\mathrm{ers}^{2}$ as an important guide to adequate but not excessive RLFP flow. In this study we have demonstrated that individual CBVI values correlate poorly with CBFV values, and therefore an independent method of measuring CBF is de-

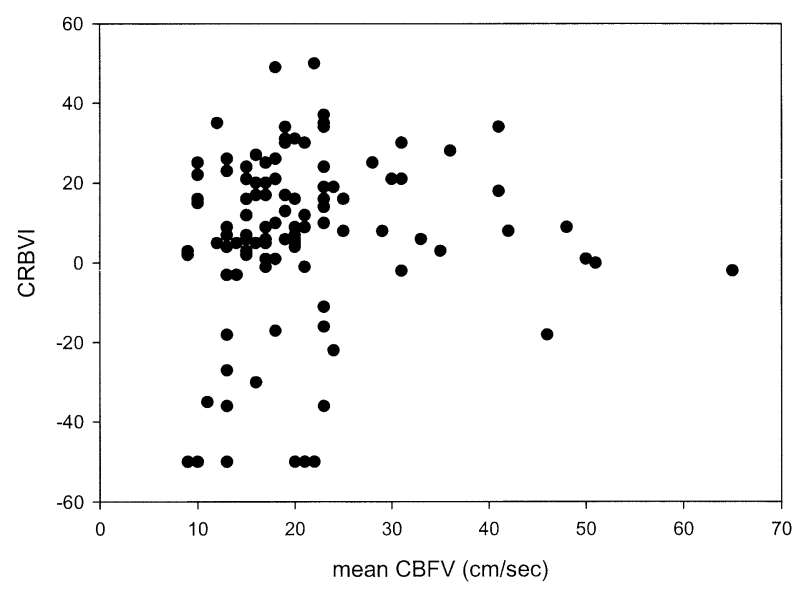

Figure 2. CBVI measured by NIRS versus mean CBFV as measured by TCD. $P>.05$ by Spearman rank order correlation.

sirable to guide RLFP flow. CBVI is actually an index of signal strength detected by the infrared light detectors. The signal strength for NIRS may be affected by factors other than blood volume, such as ambient light, interference with signal detection by darkly pigmented skin or hair, and improper application of the sensor (Somanetics INVOS 5100 Operations Manual; Somanetics Corp, Troy, Mich). Thus the designation CBVI may be misleading and in fact is not a measurement approved by the US Food and Drug Administration. On the other hand, CBFV in the middle cerebral artery has demonstrated strong correlations with CBF measured by thermodilution in adults ${ }^{14}(r=0.83, P>$ .0001 ), and with bypass pump flow (a surrogate for CBF) in children $^{15}(r=0.73, P>.001)$.

There are several limitations to this study. First, the significance of "low" $\mathrm{rSO}_{2} \mathrm{i}$ values and of a $10 \%$ or greater difference between cerebral hemispheres in neonates undergoing cardiac surgery is unknown. Austin and colleagues ${ }^{16}$ found that an $\mathrm{rSo}_{2} \mathrm{i}$ decrease of more than $20 \%$ (from a baseline established just before aortic cannulation) sustained for 3 minutes or longer in infants and children undergoing congenital heart surgery with cardiopulmonary bypass resulted in an incidence of acute postoperative neurologic morbidity (seizure, cerebral infarct, or choreoathetosis) of $26 \%$, versus an incidence of $7 \%$ if cerebral desaturation did not occur. Kurth and coworkers, ${ }^{17}$ in a study of 26 infants and children undergoing DHCA for cardiac surgery, found acute neurologic morbidity in the 3 patients (coma in 2 and seizures in 1) whose cerebral oxygen saturation failed to increase during cooling on bypass, as is the norm. The patients whose cerebral oxygen saturation did increase (mean 30\% above baseline) had no detectable neurologic morbidity. To date, this is the only evidence that low $\mathrm{rSO}_{2} \mathrm{i}$ values lead to adverse outcomes in congenital heart surgery. Although encouraging, the brief neurologic follow-up with 
standard clinical examination in our patients does not prove the adequacy of RLFP to preserve long-term central nervous system function.

We made the following assumptions in hypothesizing that the CBVI would correlate with the CBFV about the relationships between $\mathrm{CBV}$, CBVI, $\mathrm{CBF}$, and CBFV during RLFP: CBVI $\propto \mathrm{CBV} \propto \mathrm{CBF} \propto \mathrm{CBFV}$. CBVI should correlate with $\mathrm{CBV}$ if the total hemoglobin and the near infrared light path are not changing. Correlation between RLFP flow rate (CBF) and CBVI has been demonstrated by Pigula and coworkers. ${ }^{2}$ Additionally, we are assuming that CBFV is directly proportional to CBF in this setting. This is borne out by a clinical study of low-flow bypass in 28 neonates ${ }^{18}$ in which bypass flows of 10 to $50 \mathrm{~mL} /(\mathrm{kg} \cdot \mathrm{min})$ exhibited a positive correlation with CBFV. This assumption depends on an unchanging diameter of the middle and anterior cerebral arteries during different measurement conditions. This has been shown to be the case for a wide range of $\mathrm{PaCO}_{2}$ values and cerebral perfusion pressures. ${ }^{19,20}$ The $\alpha$-receptor blockade and $\mathrm{pH}$-stat blood gas management used in all our patients should also have ensured maximal vasodilation of the cerebral blood vessels. We were careful to make measurements at the same temperature and $\mathrm{PaCO}_{2}$ values to minimize any changes in the vascular resistance of the downstream cerebral vasculature. In addition, we insonated the anterior and middle cerebral arteries at the same angle to minimize variations in the angle of incidence of the pulsed-wave ultrasound, which could affect CBFV measurement. Even with all of these factors, CBFV may not directly reflect actual CBF in the individual patient, and this could potentially account for the apparent discrepancy we found in those patients who had a lower $\mathrm{rSO}_{2} \mathrm{i}$ on the left even though we measured the same or even increased $\mathrm{CBFV}$ on the same side. Indeed, our measurements demonstrated no correlation between RLFP flow rate and CBFV during RLFP.

In conclusion, RLFP provides comparable delivery of oxygenated blood to both cerebral hemispheres during neonatal aortic arch reconstruction. CBVI, as measured by NIRS, has poor correlation with CBFV measured by TCD, suggesting that TCD is useful in addition to NIRS as a guide to RLFP flow rates.

We thank Debora East, RN, for assistance with data collection, and E. O'Brian Smith, PhD, for statistical consultation.

\section{References}

1. Pigula FA, Siewers RD, Nemoto EM. Regional perfusion of the brain during neonatal aortic arch reconstruction. J Thorac Cardiovasc Surg. 1999;117:1023-4.
2. Pigula FA, Nemoto EM, Griffith BP, Siewers RD. Regional low-flow perfusion provides cerebral circulatory support during neonatal aortic arch reconstruction. J Thorac Cardiovasc Surg. 2000;119:331-9.

3. Tweddell JS, Hoffman GM, Mussatto KA, Fedderly RT, Berger S, Jacquiss RD, et al. Improved survival of patients undergoing palliation of hypoplastic left heart syndrome: lessons learned from 115 consecutive patients. Circulation. 2002;106(Suppl I):I82-9.

4. Andropoulos DB, Stayer SA, McKenzie ED, Fraser CD. Novel cerebral physiologic monitoring to guide low-flow cerebral perfusion during neonatal aortic arch reconstruction. $J$ Thorac Cardiovasc Surg. 2003;125:491-9.

5. de Vries JW, Hoorntje T, Bakker PF. Cerebral oxygen saturation monitoring in an infant undergoing ICD implantation. $J$ Cardiothorac Vasc Anesth. 1998;12:442-4.

6. Yoshitani K, Kawaguchi M, Tatsumi K, Kitaguchi K, Furuya H. A comparison of the INVOS 4100 and the NIRO 300 near-infrared spectrophotometers. Anesth Analg. 2002;94:586-90.

7. Brun NC, Moen A, Borch K, Suagstad OD, Griesen G. Near-infrared monitoring of cerebral tissue oxygen saturation and blood volume in newborn piglets. Am J Physiol. 1997;273(2 Pt 2):H682-6.

8. Fischer AQ, Truemper EJ. Applications in the neonate and child. In: Babikian VL, Wechsler LR, editors. Transcranial Doppler ultrasonography. St Louis: Mosby; 1993. p. 355-75.

9. Alpers BJ, Berry RG, Paddison RM. Anatomical studies of the circle of Willis in normal brain. Arch Neurol Psychol. 1959;81:408-18.

10. Truemper EJ, Fischer AZ. Cerebrovascular developmental anatomy and physiology in the infant and child. In: Babikian VL, Wechsler LR, editors. Transcranial Doppler ultrasonography. St Louis: Mosby; 1993. p. 303-54.

11. Mitchell DG, Merton DA, Mirsky PJ, Needleman L. Circle of Willis in newborns: color Doppler imaging of 53 healthy full-term infants. Radiology. 1989;172:201-5.

12. Watzman HM, Kurth CD, Montenegro LM, Rome J, Steven JM, Nicolson SC. Arterial and venous contributions to near-infrared cerebral oximetry. Anesthesiology. 2000;93:947-53.

13. Janelle GM, Mnookin S, Gravenstein N, Martin TD, Urdaneta F. Unilateral cerebral oxygen desaturation during emergent repair of a DeBakey type 1 aortic dissection: potential aversion of a major catastrophe. Anesthesiology. 2002;96:1263-5.

14. van der Linden J, Wesslen O, Ekroth R, Tyden H, von Ahn H. Transcranial Doppler-estimated versus thermodilution-estimated cerebral blood flow during cardiac operations. Influence of temperature and arterial carbon dioxide tension. J Thorac Cardiovasc Surg. 1991; 102:95-102.

15. van der Linden J, Priddy R, Ekroth R, Lincoln C, Pugsley W, Scallan $\mathrm{M}$, et al. Cerebral perfusion and metabolism during profound hypothermia in children. A study of middle cerebral artery ultrasonic variables and cerebral extraction of oxygen. $J$ Thorac Cardiovasc Surg.. 1991;102:103-14.

16. Austin EH, Edmonds HL, Auden SM, Seremet V, Niznik G, Sehic A, et al. Benefit of neurophysiologic monitoring for pediatric cardiac surgery. J Thorac Cardiovasc Surg. 1997;114:707-15.

17. Kurth CD, Steven JM, Nicolson SC. Cerebral oxygenation during pediatric cardiac surgery using deep hypothermic circulatory arrest. Anesthesiology. 1995;82:74-82.

18. Zimmerman AA, Burrows FA, Jonas RA, Hickey PR. The limits of detectable cerebral perfusion by transcranial Doppler sonography in neonates undergoing deep hypothermic low-flow cardiopulmonary bypass. J Thorac Cardiovasc Surg. 1997;114:594-600.

19. Aaslid R, Markwalder TM, Nornes H. Noninvasive transcranial Doppler ultrasound recording of flow velocity in basal cerebral arteries. J Neurosurg. 1982;57:769-74.

20. Volpe JJ, Perlman JM, Hill A, McMenamin JB. Cerebral blood velocity in the human newborn: the value of its determinations. Pediatrics. 1982;70:147-52. 